\title{
Nanophotonic Devices for Optical Networks-On-Chip
}

\author{
D. Van Thourhout ${ }^{1}$, I. O’'Connor ${ }^{2}$, A. Scandurra ${ }^{3}$, L. Liu ${ }^{1}$, W. Bogaerts ${ }^{1}$, S. Selvaraja ${ }^{1}$, G. Roelkens ${ }^{1}$ \\ ${ }^{1}$ Ghent University-IMEC, Department of Information Technology (INTEC), St. Pietersnieuwstraat 41, 9000 Gent, Belgium \\ ${ }^{2}$ STMicroelectronics, Catania, Italy \\ ${ }^{3}$ Lyon Insitute of Nanotechnology, Ecole Centrale de Lyon, France \\ dries.vanthourhout@ugent.be
}

\begin{abstract}
We describe an optical network-on-chip built from passive wavelength routing circuits and tunable micro transmitters based on microdisk sources. Operation of the different subcomponents will be demonstrated.

(C) Optical society of America

OCIS codes: 130.3120 Integrated optics devices ; 250.5960 Semiconductor lasers
\end{abstract}

\section{Introduction}

The use of silicon waveguides for building on-chip optical networks has attracted a lot of attention recently [1]. We showed that such an optical network-on-chip (ONoC) can be built using wavelength routing [2]: the central routing structure is kept passive, while tunable microsources are used for directing the light through the network. If these are combined with resonant detectors, collisions are avoided and latency in the routing is reduced to a minimum.
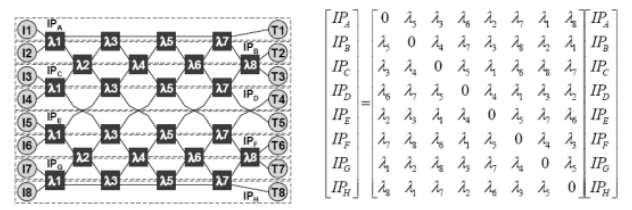

(a)
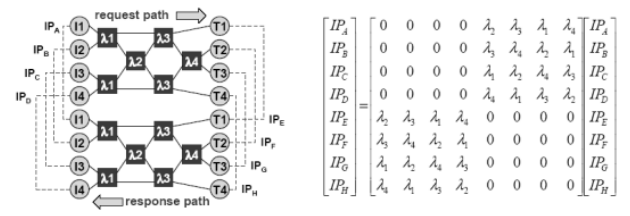

(b)

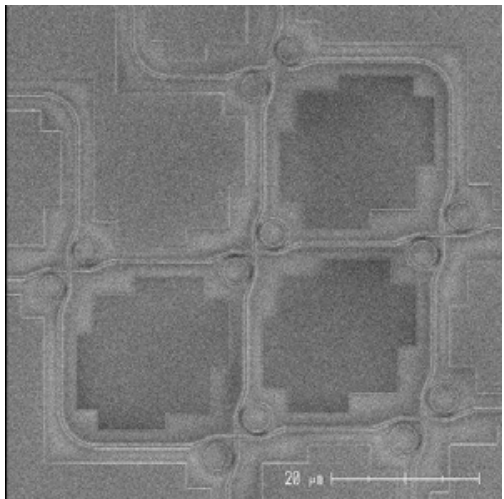

Fig. 1 Left: Communication scenarios and corresponding connectivity matrices for ONoC in 8-IP block scenarios (a) single 8x8 ONoC for total connectivity between 8 IP blocks (b) $24 \times 4$ ONoCs for request/response connectivity between 2 groups of 4 IP blocks [2]. Right: Fabricated $4 \times 4$ ONoC.

\section{Passive wavelength routing structures}

The central wavelength routing circuit can be built up from individual single wavelength routing cells. Fig. 1 (right) shows such a $4 \times 4$ wavelength routing network. However, in many cases full connectivity is not required, and the number of cells in the router can be reduced drastically (Fig. 1 left) by eliminating redundant cells.
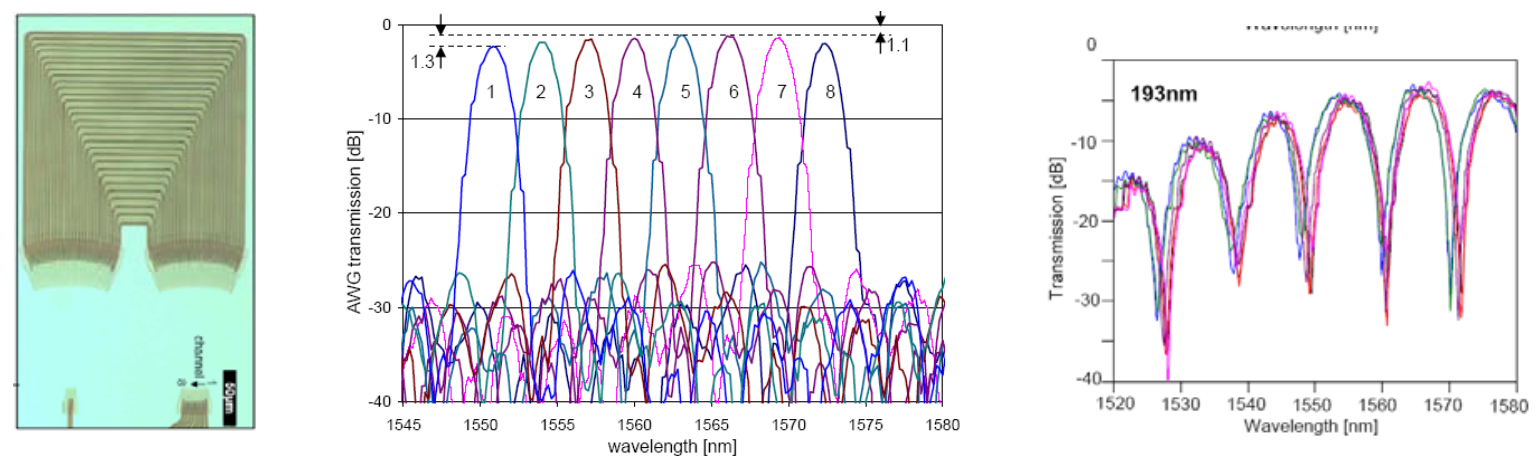

Fig. 2 a) AWG based silicon router. b) Response of 8-channel AWG. c) Fabrication uniformity of Mach Zehnder based filters.

The drawback of ring resonator structures is there extreme sensitivity to fabrication tolerances making individual tuning/trimming of the rings necessary. As an alternative, more classical AWG-based routing structures, which also 
provide full connectivity (Fig. 2a,b) can be used. Although they are slightly larger than ring based structures, a constant channel spacing is intrinsically guaranteed through the design. We have investigated fabrication tolerances using DUV248nm and 193nm mask based lithography in detail (Fig. 2c).

\section{Versatile microdisk sources}

The second essential component of the $\mathrm{ONoC}$ is the tunable transmitter. We are investigating the use of microdisk multiwavelength sources [4][5] for this purpose. As an alternative, the microdisk laser can be used as a modulator, in combination with an off-chip laser source [6]. In [7] we demonstrated all-optical wavelength conversion using microdisk sources, which may be important for scaling these networks to higher node numbers.

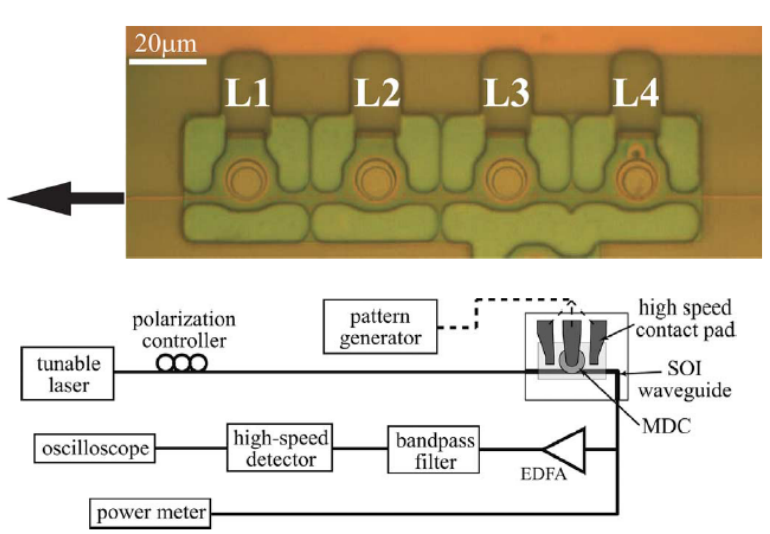

(a)

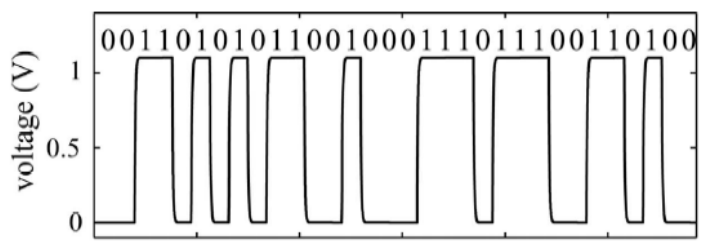

(b)

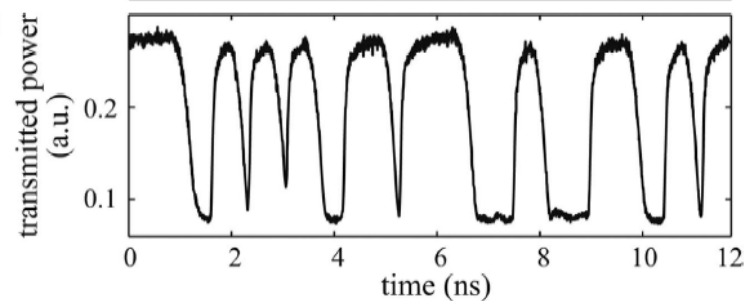

Fig. 3 Left: 4-wavelength microdisk array (top) and setup for microdisk based modulator experiment (bottom). Right: Waveform of a 32-bit NRZ signal at 2.73 Gbps applied on (a) proposed modulator and (b) corresponding optical signal [6].

\section{Conclusion}

We demonstrated that an optical NoC can be built using passive silicon routing structures, microtransmitters based on microdisk sources and wavelength selective detectors.

We acknowledge support of the EU through the FP7 project WADIMOS.

\section{References}

[1] A. Shacham, K. Bergman, L. P. Carloni, "On the Design of a Photonic Network-on-Chip," NOCS 2007, paper 2.1 (May 2007)

[2] Alberto Scandurra, Ian OConnor, 'Scalable CMOS-compatible photonic routing topologies for versatile networks on chip ', NoCArc 2008, Como (Italy)

[3] W. Bogaerts, P. Dumon, S. Selvaraja, D. Van Thourhout, R. Baets, Silicon Nanophotonic Waveguide Circuits and Devices,Leos Annual Meeting,Newport Beach, United States, p.TuU1 (2008)

[4] J. Van Campenhout, P. Rojo Romeo, P. Regreny, C. Seassal, D. Van Thourhout, S. Verstuyft, L. Di Cioccio, J.-M. Fedeli, C. Lagahe, R. Baets, Electrically pumped InP-based microdisk lasers integrated with a nanophotonic silicon-on-insulator waveguide circuit,Optics Express, 15(11), p.6744-6749 (2007)

[5] J. Van Campenhout, L. Liu, Pedro Rojo Romeo, D. Van Thourhout, Christian Seassal, Philippe Regreny, Lea Di Cioccio, Jean-Marc Fedeli, R. Baets, A Compact SOI-Integrated Multiwavelength Laser Source Based on Cascaded InP Microdisks,IEEE PHOTONICS TECHNOLOGY LETTERS, 20(16), p.1345-1347 (2008)

[6] L. Liu, J. Van Campenhout, G. Roelkens, Richard A. Soref, D. Van Thourhout, Pedro Rojo-Romeo, Philippe Regreny, Christian Seassal, Jean-Marc Fédéli, R. Baets, Carrier-injection-based electro-optic modulator on silicon-on-insulator with a heterogeneously integrated III-V microdisk cavity,Optics Letters, 33(21), p.2518 (2008)

[7] L. Liu, J. Van Campenhout, G. Roelkens, D. Van Thourhout, Pedro Rojo Romeo, Philippe Regreny, Christian Seassal, Jean-Marc Fédéli, R. Baets, Ultralow-power all-optical wavelength conversion in a silicon-on-insulator waveguide based on a heterogeneously integrated III-V microdisk laser,Applied Physics Letters, 93(6), p.061107 (2008) 